\title{
Le savoir
}

\section{The Knowledge}

\author{
E. Bories \\ (C) Lavoisier SAS 2015
}

« Le savoir est l'arme la plus efficace contre les tyrans. La preuve : ils brûlent toujours tous les livres » Erik Orsenna

La dernière édition de Vidéo-Digest a été une grande réussite, avec une participation médicale et infirmière en hausse. Les séances d'examens en direct, ciblés sur des actes de notre pratique quotidienne mais également de traitement endoscopiques plus novateurs, ont été, nous l'espérons, formateurs et motivants pour la grande majorité d'entre vous. Cette forme d'enseignement, comme nous l'avions évoqué dans des numéros précédents, constitue une voie d'amélioration de nos pratiques en endoscopie, sous réserve d'un respect strict de règles éthiques et organisationnelles. Nous espérons vous retrouver encore plus nombreux l'an prochain lors de notre congrès annuel du 3 au 5 novembre 2016.

Je ne reviendrai pas sur les tristes événements qui sont survenus le 13 novembre à Paris et qui ont conduit à annuler la dernière journée de formation. Il faut espérer que le savoir triomphera de l'obscurantisme, la fraternité de la barbarie.

Dans ce numéro d'Acta Endoscopica, vous pourrez lire deux intéressantes recommandations concernant la prise en charge des hémorragies digestives basses [1] et l'endoscopie en pédiatrie [2].

La gestion diagnostique de ces hémorragies est synthétisée sous la forme d'un algorithme simple permettant de mieux hiérarchiser les différents examens. Les progrès de l'imagerie n'effacent pas le rôle prépondérant de la coloscopie dans cette situation avec une rentabilité diagnostique élevée si elle est réalisée dans les quarante-huit premières heures. L'hémorragie diverticulaire, cause prépondérante dans les hémorragies communautaires et hospitalières, n'est identifiée que dans 6 à $42 \%$ des cas par l'endoscopie, la sensibilité étant améliorée lorsqu'elle est réalisée précocement $[3,4]$. La stratégie thérapeutique des hémorragies diver- ticulaires n'est pas standardisée, reposant essentiellement sur les traitements endoscopiques par mise de clips, ligature élastique voire injection de sérum adrénaliné $[5,6]$. Que le saignement initial se tarisse spontanément ou après traitement endoscopique, le risque de récidive précoce et retardé est variable dans la littérature, jusqu'à $50 \%$, l'effet du traitement endoscopique restant incertain [7].

Promise lors du précédent numéro mais décalée pour des problèmes éditoriaux, vous trouverez enfin la recommandation de la commission pédiatrie sur endoscopie et MICI de l'enfant très enrichissante.

Enfin, un article original revient sur les interactions bénéfiques ou néfastes de la pratique sportive avec les pathologies digestives.

En vous souhaitant une bonne lecture !

\section{Références}

1. Ah-Soune P, Barthet M. Hémorragie digestive basse : algorithme de prise en charge. Acta Endoscopica 2015;45:321-4

2. Lachaux A. Endoscopie digestive et maladies inflammatoires chroniques de l'intestin en pédiatrie. Acta Endoscopica 2015;45:325-30

3. Strate LL, Syngal S. Predictors of utilization of early colonoscopy vs radiography for severe lower intestinal bleeding. Gastrointest Endosc 2005;61:46-52

4. Niikura R, Nagata N, Aoki T, et al. Predictors for identification of stigmata of recent hemorrhage on colonic diverticula in lower gastrointestinal bleeding. J Clin Gastroenterol 2015;49:e24-e30

5. Kaltenbach T, Watson R, Shah J, et al. Colonoscopy With Clipping Is Useful in the Diagnosis and Treatment of Diverticular Bleeding. Clin Gastroenterol Hepatol 2012;10:131-7

6. Farrell JJ, Graeme-Cook F, Kelsey PB. Treatment of bleeding colonic diverticula by endoscopic band ligation: an in-vivo and ex-vivo pilot study. Endoscopy 2003;35:823-9

7. Nakano K, Ishii N, Ikeya T, et al. Comparison of long-term outcomes between endoscopic band ligation and endoscopic clipping for colonic diverticular hemorrhage. Endosc Int Open 2015;3: E529-33
E. Bories $(\bowtie)$

Unité d'exploration médicochirurgicale oncologique,

Institut Paoli-Calmettes, 232 boulevard de Sainte-Marguerite,

F-13273 Marseille cedex 09

e-mail : boriese@ipc.unicancer.fr 